\title{
Adequação do pré-natal e peso ao nascer: uma revisão sistemática
}

\author{
Adequacy of prenatal care and birthweight: \\ a systematic review
}

\author{
1 Departamento de Medicina \\ Social, Faculdade de \\ Medicina, Universidade \\ Federal de Pelotas, \\ Pelotas, Brasil. \\ Correspondência \\ D. S. Silveira \\ Departamento de Medicina \\ Social, Faculdade de \\ Medicina, Universidade \\ Federal de Pelotas. \\ Av. José Maria da Fontoura \\ 1218, Pelotas, RS \\ 96090-370, Brasil. \\ denisilveira@uol.com.br
}

\section{Abstract}

This was a systematic literature review on publications in which prenatal care was investigated as a predictive factor for birthweight. The MEDLINE, Cochrane Library, and SciELO databases were searched using a combination of the following uniterms: "prenatal care", "antenatal care", "quality", "adequacy", "birthweight”, and "low birthweight". Twenty-five studies were found: seventeen had a cross-sectional design, in addition to four cohort studies, three casecontrol studies, and one randomized trial. The adequacy indicators related to utilization (quantitative measures) and content of prenatal care (process or qualitative indicators). Most authors employed quantitative indicators, mainly the Kessner Index and the Adequacy of Prenatal Care Utilization Index. Qualitative criteria were used in only two studies. Most of the cross-sectional studies found a protective effect of prenatal care against low birthweight, whereas results of studies with other designs were conflicting. This review's findings highlight that the impact of prenatal care on birthweight is not unequivocal, mainly due to the effect of self-selection bias. Randomized trials are needed to elucidate such a relationship.

Prenatal Care; Birth Weight; Quality; Review Literature
Denise S. Silveira 1

Iná S. Santos 1

\section{Introdução}

O cuidado pré-natal compreende um conjunto de atividades no transcurso da gravidez que requerem tempo e outros investimentos tanto por parte da mulher, como de profissionais e de organizações que se dedicam à oferta desse cuidado. A atenção pré-natal é utilizada como um indicador de boa prática, assim como uma medida de qualidade de cuidados clínicos e de saúde pública 1,2 .

Em contraste com a similaridade dos objetivos do atendimento pré-natal, há ainda uma enorme variação na prática dessa atenção entre países, comunidades e instituições, o que resulta em questionamentos sobre a efetividade e segurança dos vários elementos que o constituem 3,4 .

Portanto, muitas questões quanto à freqüência, à especificidade das intervenções envolvidas e ao seu conteúdo ainda são objeto de discussão, na tentativa de definição de padrões, tais como o do momento adequado de iniciar as consultas e sua periodicidade e a introdução de conhecimentos médicos e tecnologias, com a adequada validação científica 5,6,7.

Em geral, as investigações da atenção ao pré-natal têm como objetivo subsidiar ações de planejamento, monitorar a tendência na utilização de cuidados e entender a sua relação com resultados 8,9 . Os fatores geralmente incluídos nos estudos epidemiológicos procuram medir exposições econômicas, o uso de tecno- 
logia apropriada e a qualidade da atenção, essa última abrangendo principalmente marcadores de acesso, de utilização, de cobertura, de eficácia, do alcance de objetivos, da estrutura dos serviços, do processo de atendimento, da satisfação do usuário e de resultados da atenção 10.

Entre os indicadores empregados para o estudo da adequação do cuidado pré-natal, evidenciam-se dois grupos distintos: os de utilização ou quantitativos; e os de conteúdo ou de processo ou qualitativos. A maioria das investigações se concentra no uso dos indicadores quantitativos, através de informações sobre a idade gestacional de início do cuidado e o número de consultas de pré-natal, isoladas ou combinadas, controlados ou não para a duração da gestação ${ }^{11}$. As medidas combinadas mais freqüentemente escolhidas são o Índice de Kessner e o APNCUI (Adequacy Prenatal Care Utilization Index) ou Índice de Kotelchuck 9,12,13.

O Índice de Kessner é um algoritmo baseado no trimestre em que o cuidado pré-natal se inicia e no número de consultas, ajustado para a idade gestacional, sendo definido por três categorias de utilização: pré-natal adequado, intermediário e inadequado.

O APNCUI caracteriza a utilização do pré-natal em duas dimensões independentes e distintas: a adequação início do cuidado pré-natal agrupada em quatro categorias, e a adequação da atenção recebida, baseada no número esperado de consultas, de acordo com o American College of Obstetricians and Gynecologists (ACOG), para gestações não complicadas, também em quatro grupos. A razão entre o observado e o esperado categoriza a utilização em sobreadequada, adequada, intermediária e inadequada.

Poucos estudos exploram a qualidade do cuidado, que pode ser expressa pela performance apropriada de intervenções e exige a comparação com um padrão, muitas vezes, não definido de consenso, dificultando a operacionalização das investigações e a comparação dos achados 14,15,16.

O crescente aumento da disponibilidade e utilização do cuidado pré-natal têm sido associados à melhora do bem-estar da mãe e da criança e conseqüente redução de resultados perinatais adversos 3,9,14,17,18. Nesse contexto, um dos indicadores de resultado mais estudados é o peso ao nascer, pois, além de ser o principal determinante da mortalidade infantil, é uma medida obtida com facilidade e confiança a partir de registros de nascimentos 4 , $12,13,19,20,21$.
Este estudo é uma revisão bibliográfica das publicações epidemiológicas que investigaram a relação do cuidado pré-natal com o peso ao nascer.

\section{Metodologia}

Foram rastreados estudos, realizados entre 1990 e 2002, no Brasil ou exterior, em que a atenção pré-natal foi uma das variáveis independentes de interesse na análise de resultados da gravidez, especificamente, o peso ao nascer.

As bases de dados empregadas para o rastreamento dos artigos foram MEDLINE, Cochrane Library e SciELO, utilizando-se a combinação das palavras-chave "prenatal care", "antenatal care", "quality”, “adequacy”, "birthweight” e "low birthweight". Investigou-se também a lista de referência dos estudos selecionados.

Incluíram-se estudos epidemiológicos em humanos, publicados em inglês, português ou espanhol. Todos os resumos de artigos contendo as palavras-chave foram lidos, inclusive os identificados das listas de referências, e considerados relevantes aqueles com relato da aplicação de indicadores de utilização e/ou conteúdo no estudo das associações do pré-natal com peso ao nascer.

Os critérios para a classificação da adequação do pré-natal não foram definidos antecipadamente, principalmente por não existir um padrão de consenso. Portanto, todas as definições propostas pelos investigadores foram consideradas. Procedeu-se a um primeiro rastreamento incluindo apenas a combinação das palavras-chave "prenatal care", "antenatal care", "quality" e "adequacy", duas a duas, no qual buscou-se identificar as duplicatas de resumos, excluir as referências de artigos sem resumo disponível e aqueles não especificamente relacionados ao tema de interesse.

Em um terceiro momento, foram selecionadas somente as publicações de estudos que investigaram peso ao nascer ou baixo peso ao nascer (BPN: peso ao nascer inferior a $2.500 \mathrm{~g}$ ) como resultado da atenção pré-natal.

\section{Resultados}

Um total de 229 artigos foi obtido por meio do primeiro rastreamento (Tabela 1). Dos 25 estudos que atenderam ao critério de utilização do peso ao nascer, como desfecho, 17 eram trans- 
Tabela 1

Resultado da busca de artigos sobre cuidado pré-natal e peso ao nascer.

\begin{tabular}{lcrr}
\hline Pesquisa & 1ạ etapa & 2a etapa & Peso ao nascer \\
\hline Prenatal care/Quality & 576 & 205 & 20 \\
Prenatal care/Adequacy & 189 & 22 & 3 \\
Antenatal care/Quality & 10 & 4 & 2 \\
Antenatal care/Adequacy & 2 & 0 & 0 \\
Total & 775 & 229 & 25 \\
\hline
\end{tabular}

versais, quatro coortes, três caso-controle e um ensaio clínico. A Tabela 2 apresenta uma sinopse das publicações revisadas.

Todos os estudos que utilizaram o peso de nascimento categorizado foram unânimes na definição de BPN (<2.500g) e de muito baixo peso ao nascer (MBPN: peso ao nascer inferior a $1.500 \mathrm{~g}$ ). O peso ao nascer foi analisado como variável contínua em três artigos 4,22,23.

A maioria das publicações incluiu indicadores de utilização do pré-natal, isolados ou combinados, modificados ou não. Entre esses, os indicadores mais empregados foram os Índices de Kessner 19,24,25,26,27,28 e o APNCUI 20,28,29,30,31.

Outros indicadores quantitativos usados foram: o número de consultas pré-natais, a idade de início do acompanhamento pré-natal, o número de visitas domiciliares, categorias de adequação definidas pelos autores do estudo e fazer ou não pré-natal. Três artigos empregaram, na análise, dois indicadores de utilização, o de Kessner e o APNCUI 13,20,32.

Somente dois estudos utilizaram indicadores qualitativos através de critérios próprios, que incluíam procedimentos clínicos e aconselhamento materno 4,8 .

A maior parte das publicações ajustou o efeito do cuidado pré-natal para variáveis de confusão. Os fatores de confusão maternos considerados mais freqüentemente foram: idade; escolaridade; renda; estado civil; raça/etnia; consumo de fumo, álcool ou drogas na gestação; paridade; morbidades obstétricas e fatores nutricionais, tais como índice de massa corporal, peso pré-gestacional e ganho de peso materno. História de recém-nascido com BPN em gestações prévias foi considerada em três estudos 8,20,32. A altura materna foi controlada apenas em uma investigação 33, e características do recém-nascido, tais como sexo e idade gestacional, foram consideradas por Wright 33 e Peabody 4 . Apenas quatro estudos realizaram análise ajustada para variáveis geográficas 4,27,31,34, e três, para fatores dos serviços de saúde 4,23,26.

\section{Utilização do pré-natal e peso ao nascer}

Tomados em conjunto, os estudos que analisaram a associação entre utilização do pré-natal e peso ao nascer mostraram que o cuidado adequado, ajustado para variáveis de confusão, protegia contra os nascimentos de baixo peso. Quando os indicadores Kessner e APNCUI foram aplicados no mesmo estudo, observou-se que ambos os critérios eram concordantes quanto ao risco decrescente de baixo peso no sentido do inadequado para o adequado. A última categoria de adequação do APNCUI (sobreadequação), no entanto, geralmente revelava maior risco de BPN para recém-nascidos de mães que foram a $110 \%$ ou mais das consultas recomendadas para sua idade gestacional de início do pré-natal (como o índice não ajusta para condições de risco da mãe, mulheres que consultaram mais poderiam ser aquelas com gestações complicadas).

Das quatro coortes, apenas duas detectaram efeito protetor da adequação do pré-natal sobre o menor peso de nascimento 28,29 .

Entre os três estudos caso-controle, um observou resultado de risco da atenção pré-natal inadequada para menor peso ao nascer pelo Índice de Kessner 20; o outro observou resultado também de risco para BPN, porém da categoria pré-natal sobreadequada pelo APNCUI 30 ; e o terceiro, que aplicou um escore qualitativo do conteúdo das consultas como critério de adequação, não mostrou associação estatisticamente significativa ${ }^{8}$. Exceto um 31 , todos os estudos transversais mostraram efeito protetor do pré-natal adequado contra o menor peso ao nascer.

\section{Conteúdo do pré-natal e peso ao nascer}

Dos dois estudos que empregaram o conteúdo do cuidado pré-natal como uma variável independente sobre o peso ao nascer, apenas um detectou efeito protetor dos procedimentos clínicos 4 . Nesse mesmo estudo, foram avaliados os efeitos do aconselhamento geral e específico para gestantes em risco, não sendo também detectado efeito estatisticamente significativo sobre o peso ao nascer.

\section{Discussão}

As avaliações em saúde são necessárias para estabelecer níveis e tendências de resultados específicos, identificar suas características e determinantes, assim como para avaliar a efetividade de programas destinados a influenciar 
Estudos que investigaram a relação peso ao nascer e adequação do cuidado pré-natal.

\begin{tabular}{|c|c|c|c|c|c|c|}
\hline Estudo & Delineamento & Amostra & $\begin{array}{l}\text { Definição } \\
\text { do desfecho }\end{array}$ & Critério de adequação & Fatores de confusão & Principais resultados \\
\hline Alexy et al. 43 & Transversal & 779 mulheres & BPN & $\begin{array}{l}\text { Idade gestacional } \\
\text { da } 1 \text { a consulta } \\
\text { de pré-natal e } \\
\text { no de consultas }\end{array}$ & $\begin{array}{l}\text { Maternos: idade, } \\
\text { escolaridade, renda } \\
\text { familiar, raça, tipo de } \\
\text { alimentação }\end{array}$ & $\begin{array}{l}\text { < BPN para início precoce } \\
\text { do pré-natal e maior } \\
\text { número de consultas }\end{array}$ \\
\hline Balcazar et al. 44 & Transversal & $\begin{array}{l}101.206 \text { registros } \\
\text { de nascimento }\end{array}$ & BPN & $\begin{array}{l}\text { Idade gestacional da } 1 \underline{a} \\
\text { consulta de pré-natal, } \\
\text { no de consultas e } \\
\text { duração da gestação } \\
\text { em } 6 \text { categorias }\end{array}$ & $\begin{array}{l}\text { Maternos: raça/etnia, } \\
\text { idade, risco gestacional, } \\
\text { local de moradia }\end{array}$ & $\begin{array}{l}<\text { BPN em pré-natal } \\
\text { adequado }\end{array}$ \\
\hline Barros et al. 26 & Transversal & 3.734 mulheres & BPN & Kessner & $\begin{array}{l}\text { Recém-nascido: sexo } \\
\text { e idade gestacional } \\
\text { no nascimento } \\
\text { Maternos: idade, estado } \\
\text { civil, uso de fumo, } \\
\text { complicações e ganho } \\
\text { de peso na gestação, } \\
\text { classe social } \\
\text { Serviço de saúde: tipo } \\
\text { de hospital do parto }\end{array}$ & $\begin{array}{l}\text { BPN e pré-natal adequado } \\
\text { OR } 0,39(0,23-0,65) \\
\text { BPN e pré-natal } \\
\text { intermediário } \\
\text { OR } 0,47(0,29-0,76)\end{array}$ \\
\hline Brabin et al. 45 & Transversal & $\begin{array}{l}615 \\
\text { adolescentes }\end{array}$ & BPN & $\begin{array}{l}\text { № de consultas em } \\
4 \text { categorias }\end{array}$ & $\begin{array}{l}\text { Maternos: idade, } \\
\text { escolaridade e renda } \\
\text { familiar }\end{array}$ & $\begin{array}{l}\text { Menor proporção de BPN } \\
\text { quanto maior o número } \\
\text { de consultas }(p=0,01)\end{array}$ \\
\hline $\begin{array}{l}\text { Bradley \& } \\
\text { Martin } 22\end{array}$ & Transversal & 381 mulheres & $\begin{array}{l}\text { Peso ao } \\
\text { nascer }\end{array}$ & $\begin{array}{l}\text { Care coordination } \\
\text { adequacy-index pelo no } \\
\text { de consultas domiciliares }\end{array}$ & $\begin{array}{l}\text { Maternos: idade, } \\
\text { escolaridade e renda } \\
\text { familiar }\end{array}$ & $\begin{array}{l}\text { Peso ao nascer } 162 \mathrm{~g} \text { maior } \\
\text { no cuidado pré-natal } \\
\text { intermediário }\end{array}$ \\
\hline $\begin{array}{l}\text { Collins \& } \\
\text { David } 25\end{array}$ & Transversal & $\begin{array}{l}93.101 \text { registros } \\
\text { de nascimento }\end{array}$ & BPN & Kessner & $\begin{array}{l}\text { Análise estratificada por } \\
\text { renda entre mulheres } \\
\text { brancas e negras }\end{array}$ & $\begin{array}{l}<\text { BPN em pré-natal } \\
\text { adequado }\end{array}$ \\
\hline $\begin{array}{l}\text { Delgado- } \\
\text { Rodríguez } \\
\text { et al. } 20\end{array}$ & Caso-controle & $251 / 381$ & BPN & $\begin{array}{l}\text { Kessner } \\
\text { APNCUI }\end{array}$ & $\begin{array}{l}\text { Maternos: escolaridade, } \\
\text { índice de massa corporal, } \\
\text { BPN prévio, paridade e } \\
\text { nível sócio-econômico }\end{array}$ & $\begin{array}{l}\text { BPN e pré-natal inadequado } \\
\text { Kessner OR } 2,1(1,2-3,7) \\
\text { BPN e pré-natal inadequado } \\
\text { APNCUI } 1,6(0,8-3,1)\end{array}$ \\
\hline Haas et al. 21 & Transversal & $\begin{array}{l}80.537 \text { registros } \\
\text { de nascimentos }\end{array}$ & BPN & $\begin{array}{l}\text { US Public Health Service } \\
\text { Expert Panel }\end{array}$ & $\begin{array}{l}\text { Maternos: idade, } \\
\text { paridade, raça, gestação } \\
\text { múltipla, escolaridade, } \\
\text { estado civil, renda, uso } \\
\text { de fumo e álcool }\end{array}$ & $\begin{array}{l}\text { BPN e pré-natal adequado } \\
\text { pelo no de consultas } \\
\text { OR } 0,53(0,45-0,61)\end{array}$ \\
\hline Katz et al. 19 & Transversal & $\begin{array}{l}168.322 \text { registros } \\
\text { de nascimentos }\end{array}$ & BPN & Kessner & $\begin{array}{l}\text { Maternos: idade, estado } \\
\text { civil e no de gestações }\end{array}$ & $\begin{array}{l}\text { BPN Washington-Medicaid } \\
\text { OR } 1,4(1,3-1,5) \\
\text { PN inadequado do Medicaid: } \\
\text { OR } 3,2(2,9-3,4) \\
\text { BPN British-100\% subsidiado } \\
\text { OR } 1,3(1,1-1,6) \\
\text { PN inadequado do British- } \\
\text { 100\% subsidiado } \\
\text { OR } 1,5(1,3-1,7)\end{array}$ \\
\hline Kotelchuck 13 & Transversal & $\begin{array}{l}9.941 \text { registros } \\
\text { de nascimentos }\end{array}$ & BPN & $\begin{array}{l}\text { Kessner } \\
\text { APNCUI }\end{array}$ & Comparação de índices & $\begin{array}{l}\text { BPN com PN } \\
\text { sobreadequado }=38,8 \% \\
\text { (odds de BPN bruto 2,70) } \\
\text { BPN com PN inadequado = } \\
24,5 \% \text { (odds de BPN } \\
\text { bruto } 1,47 \text { ) }\end{array}$ \\
\hline
\end{tabular}


Tabela 2 (continuação)

\begin{tabular}{|c|c|c|c|c|c|c|}
\hline Estudo & Delineamento & Amostra & $\begin{array}{l}\text { Definição } \\
\text { do desfecho }\end{array}$ & Critério de adequação & Fatores de confusão & Principais resultados \\
\hline $\begin{array}{l}\text { Krueger \& } \\
\text { Scholl } 32\end{array}$ & Coorte & $\begin{array}{l}1.771 \\
\text { adolescentes }\end{array}$ & BPN & $\begin{array}{l}\text { Kessner } \\
\text { APNCUI }\end{array}$ & $\begin{array}{l}\text { Maternos: idade, raça/ } \\
\text { etnia, paridade, fumo } \\
\text { na gestação, índice de } \\
\text { massa corporal, peso } \\
\text { pré-gravídico, ganho } \\
\text { de peso na gestação } \\
\text { e BPN prévio }\end{array}$ & $\begin{array}{l}\text { BPN e inadequado Kessner } \\
\text { OR } 1.46(1,00-2,12) \\
\text { BPN e inadequado APNCUI } \\
\text { OR } 1.14(0,80-1,62) \\
\text { BPN e sobreadequado } \\
\text { APNCUI } \\
\text { OR } 1.96(1,32-2,89)\end{array}$ \\
\hline Markson et al. 27 & Transversal & $\begin{array}{l}772 \text { mulheres } \\
\text { HIV+ } \\
2.377 \text { mulheres } \\
\text { da população } \\
\text { em geral }\end{array}$ & BPN & Kessner & $\begin{array}{l}\text { Maternos: local de } \\
\text { moradia, ano do parto, } \\
\text { idade gestacional de } \\
\text { ingresso no Medicaid, } \\
\text { idade no momento } \\
\text { do parto, raça/etnia, } \\
\text { escolaridade, nível de } \\
\text { risco clínico, uso de } \\
\text { drogas ilícitas e tabagismo } \\
\text { na gestação }\end{array}$ & $\begin{array}{l}\text { BPN e pré-natal adequado } \\
\text { OR } 0,54(0,42-0,68) \text {. }\end{array}$ \\
\hline $\begin{array}{l}\text { McDuffie } \\
\text { et al. } 46\end{array}$ & $\begin{array}{l}\text { Ensaio clínico } \\
\text { randomizado }\end{array}$ & 2.764 gestantes & $\begin{array}{l}\text { BPN } \\
\text { MBPN }\end{array}$ & $\begin{array}{l}\text { No de consultas: } \\
\text { - Intervenção: } 9 \\
\text { - Controle: } 14\end{array}$ & $\begin{array}{l}\text { Grupos basais } \\
\text { comparáveis quanto à } \\
\text { raça, etnia, paridade, } \\
\text { escolaridade e idade } \\
\text { gestacional de ingresso } \\
\text { no estudo }\end{array}$ & $\begin{array}{l}\text { BPN entre os grupos } \\
\text { RR } 0,94(0,78-1,17) \\
\text { MBPN entre os grupos } \\
\text { RR } 1,08(0,65-1,79)\end{array}$ \\
\hline $\begin{array}{l}\text { Newschaffer } \\
\text { et al. } 31\end{array}$ & Transversal & $\begin{array}{l}353 \text { mulheres } \\
\text { com uso ilícito } \\
\text { de drogas na } \\
\text { gravidez }\end{array}$ & BPN & APNCUI modificado & $\begin{array}{l}\text { Maternos: local de } \\
\text { moradia, ano do parto, } \\
\text { nacionalidade, idade, } \\
\text { raça, escolaridade, } \\
\text { estado civil, fatores de } \\
\text { risco médico, grau de } \\
\text { severidade da infecção } \\
\text { HIV e momento do } \\
\text { diagnóstico da infecção } \\
\text { HIV }\end{array}$ & $\begin{array}{l}\text { BPN e pré-natal adequado } \\
\text { OR } 0,77(0,43-1,63)\end{array}$ \\
\hline Peabody et al. 4 & Transversal & $\begin{array}{l}913 \text { mulheres } \\
\text { usuárias } \\
338 \text { clínicas } \\
\text { APS }\end{array}$ & $\begin{array}{l}\text { Peso ao } \\
\text { nascer }\end{array}$ & $\begin{array}{l}\text { Procedimentos clínicos } \\
\text { da consulta PN e } \\
\text { aconselhamento }\end{array}$ & $\begin{array}{l}\text { Recém-nascido: sexo } \\
\text { Maternos: local de } \\
\text { moradia, renda per } \\
\text { capita, escolaridade } \\
\text { Serviço de saúde: } \\
\text { variáveis da estrutura } \\
\text { e processo }\end{array}$ & $\begin{array}{l}\text { Prevalência BPN } 9,8 \% \\
\text { Peso ao nascer para exame } \\
\text { clínico completo B } 128(2.8)\end{array}$ \\
\hline $\begin{array}{l}\text { Petersen } \\
\text { et al. } 47\end{array}$ & Transversal & $\begin{array}{l}131.845 \text { registros } \\
\text { de nascimentos }\end{array}$ & BPN & $\begin{array}{l}\text { Trimestre de início do } \\
\text { pré-natal e no consultas } \\
\text { pelo } \mathrm{ACOG}^{*}\end{array}$ & $\begin{array}{l}\text { Não foi realizada análise } \\
\text { ajustada }\end{array}$ & $\begin{array}{l}\text { Maior proporção de BPN } \\
\text { entre as mulheres com } \\
\text { cuidado pré-natal } \\
\text { inadequado }\end{array}$ \\
\hline Petitti et al. 8 & Caso-controle & $124 / 157$ & BPN & $\begin{array}{l}\text { Escore de qualidade do } \\
\text { conteúdo das consultas } \\
\text { (8 itens). }\end{array}$ & $\begin{array}{l}\text { Maternos: idade, } \\
\text { paridade, nível sócio- } \\
\text { econômico, uso de fumo } \\
\text { e álcool na gestação, } \\
\text { história de BPN e baixo } \\
\text { peso pré-gestacional. }\end{array}$ & $\begin{array}{l}\text { BPN e escore: associação } \\
\text { não estatisticamente } \\
\text { significativa entre as } \\
\text { mulheres brancas }(p=0,14) \\
\text { e as negras }(p=0,11)\end{array}$ \\
\hline Poland et al. 24 & Transversal & 202 mulheres & $\begin{array}{l}\text { Normal } \\
\text { BPN } \\
\text { MBPN }\end{array}$ & $\begin{array}{l}\text { Qualidade do cuidado: } \\
\text { Kessner modificado }+ \\
\text { fonte do cuidado }\end{array}$ & $\begin{array}{l}\text { Maternos: sócio- } \\
\text { demográficos, } \\
\text { experiência da gravidez } \\
\text { e do cuidado pré-natal, } \\
\text { mês de suspeita da } \\
\text { gravidez, hipertensão e } \\
\text { abuso de substâncias }\end{array}$ & $\begin{array}{l}\text { Peso ao nascer e qualidade } \\
\text { do cuidado } \beta 0,26(p<0,01)\end{array}$ \\
\hline
\end{tabular}

(continua) 
Tabela 2 (continuação)

\begin{tabular}{|c|c|c|c|c|c|c|}
\hline Estudo & Delineamento & Amostra & $\begin{array}{l}\text { Definição } \\
\text { do desfecho }\end{array}$ & Critério de adequação & Fatores de confusão & Principais resultados \\
\hline Raine et al. 29 & Coorte & 3.334 mulheres & BPN & APNCUI & $\begin{array}{l}\text { Maternos: idade, estado } \\
\text { civil, história de aborto } \\
\text { prévio, BPN prévio, } \\
\text { intervalo interpartal e } \\
\text { fumo na gestação }\end{array}$ & $\begin{array}{l}\text { BPN e pré-natal inadequado } \\
\text { OR } 1,3(0,9-2,0)\end{array}$ \\
\hline $\begin{array}{l}\text { Ramirez-Zetina } \\
\text { et al. } 23\end{array}$ & Transversal & 279 mulheres & $\begin{array}{l}\text { Peso ao } \\
\text { nascer }\end{array}$ & Fazer/não fazer pré-natal & $\begin{array}{l}\text { Conceitos, atitudes e } \\
\text { práticas maternas } \\
\text { em relação à saúde } \\
\text { obstétrica } \\
\text { Acesso a serviços } \\
\text { de saúde }\end{array}$ & $\begin{array}{l}\text { Não foram encontradas } \\
\text { crianças recém-nascidas } \\
\text { com peso }<2.500 \mathrm{~g} \\
\text { Não fazer pré-natal e maior } \\
\text { proporção de peso ao } \\
\text { nascer }<3.000 \mathrm{~g}(p<0,001)\end{array}$ \\
\hline $\begin{array}{l}\text { Shohan-Vardi } \\
\text { et al. } 30\end{array}$ & Caso-controle & $189 / 384$ & BPN & APNCUI modificado & $\begin{array}{l}\text { Idade materna e } \\
\text { paridade }\end{array}$ & $\begin{array}{l}\text { BPN e idade gestacional do } \\
\text { início do pré-natal: } \\
14-22 \text { OR } 0,99(0,63-1,54) ; \\
23 \text { ou + OR } 1,27(0,60-2,70) \\
\text { BPN e adequação: } \\
\text { Inadequado OR } 1,28 \\
(0,70-2,37) ; \\
\text { cuidado sobreadequado } \\
\text { OR } 6,10(2,09-17,78)\end{array}$ \\
\hline Silva et al. 48 & Transversal & 2.541 mulheres & BPN & $\begin{array}{l}\text { Adequação em } 3 \\
\text { categorias }\end{array}$ & $\begin{array}{l}\text { A variável da adequação } \\
\text { não foi levada para } \\
\text { análise ajustada }\end{array}$ & $\begin{array}{l}\text { BPN e pré-natal inadequado } \\
\text { OR bruta } 0,98(0,70-1,36)\end{array}$ \\
\hline Turner et al. 28 & Coorte & $\begin{array}{l}2.254 \text { mulheres } \\
\text { HIV+ }\end{array}$ & BPN & Kessner & $\begin{array}{l}\text { Maternos: idade, } \\
\text { escolaridade, raça/grupo } \\
\text { étnico, uso de fumo } \\
\text { e álcool na gestação, } \\
\text { paridade, diagnóstico } \\
\text { de AIDS antes e até } 1 \text { ano } \\
\text { após o parto }\end{array}$ & $\begin{array}{l}\text { BPN e pré-natal adequado } \\
\text { OR } 0,52(0,41-0,65)\end{array}$ \\
\hline Turner et al. 34 & Coorte & $\begin{array}{l}1.858 \text { mulheres } \\
\text { HIV+ }\end{array}$ & BPN & APNCUI modificado & $\begin{array}{l}\text { Maternos: idade, } \\
\text { raça/grupo étnico, } \\
\text { escolaridade, estado } \\
\text { civil, comorbidades } \\
\text { crônicas, uso de fumo } \\
\text { e abuso de substâncias } \\
\text { na gestação, local de } \\
\text { residência e naturalidade }\end{array}$ & $\begin{array}{l}\text { BPN e pré-natal adequado } \\
\text { OR } 0,80(0,61-1,05)\end{array}$ \\
\hline Wright 33 & Transversal & 1.597 mulheres & BPN & $\begin{array}{l}\text { Não fazer pré-natal ou } \\
\text { inadequação do pré-natal }\end{array}$ & $\begin{array}{l}\text { Recém-nascido: idade } \\
\text { gestacional e sexo } \\
\text { Maternos: idade, altura, } \\
\text { ocupação e renda familiar, } \\
\text { morbidades na gestação } \\
\text { e fatores obstétricos }\end{array}$ & $\begin{array}{l}\text { Pré-natal inadequado foi } \\
\text { responsável por } 8 \% \text { do BPN }\end{array}$ \\
\hline
\end{tabular}


esses resultados 35. Esta revisão mostrou que a maioria dos estudos que avaliaram o efeito da adequação do cuidado pré-natal sobre o peso ao nascer encontrou um resultado positivo. Tendo em vista a maior facilidade de publicarse estudos com resultados positivos, geralmente considerados mais interessantes no processo editorial, a possibilidade do viés de publicação não pode ser descartada. Assim, os artigos que realmente chegam a ser publicados são uma amostra viciada de todos os resultados de pesquisa 36,37 .

No estabelecimento da cadeia causal através da qual a atenção pré-natal adequada exerceria seu efeito sobre a prevenção do BPN, a temporalidade e a classificação de risco da gestante são variáveis essenciais. Nesse sentido, o grau de inferência causal auferida pelo desenho dos estudos precisa ser levado em conta. $\mathrm{Na}$ atual revisão, os estudos com desenhos mais robustos, além de menos numerosos (apenas oito), não foram consistentes no achado de proteção do pré-natal contra o BPN. Os achados das avaliações transversais, por outro lado, quase que invariavelmente foram positivos.

Embora esforços consideráveis ainda sejam feitos para melhorar o acesso a serviços de prénatal, as evidências ainda são controversas quando se estuda a associação do cuidado com peso ao nascer ${ }^{9}$. Parte dessa controvérsia pode ser atribuída a escolha, pela maioria dos estudos, de indicadores puramente quantitativos. Tais indicadores têm limitada sensibilidade como medida de exposição ao pré-natal, pois não medem o conteúdo do cuidado, importante elemento da qualidade, abrangência e continuidade 17,35. Além disso, os indicadores quantitativos podem ser afetados tanto por fatores individuais maternos, sócio-demográficos e crenças, quanto da organização dos serviços de saúde 19,38. É sabido, por exemplo, que o nível de escolaridade da mulher interfere no número de consultas pré-natais; que a presença de companheiro fixo influencia a idade de início do pré-natal e o número total de consultas; e que mulheres com mais filhos costumam comparecer mais tardiamente ao pré-natal 19,39.

Um outro aspecto dificultando o estabelecimento da consistência dos achados relaciona-se à heterogeneidade dos indicadores quantitativos utilizados. A classificação das mulheres com vários índices resulta em diferentes padrões de utilização do cuidado pré-natal 40 . Em conseqüência, os resultados de estudos que aplicaram indicadores diferentes não têm, necessariamente, o mesmo significado.

É importante ressaltar a escassez de estudos incluindo medidas de conteúdo do cuida- do pré-natal. A diversidade de protocolos afeta a implementação de pesquisas, os resultados dos estudos e dificulta comparações 8,11,19. Outro problema surge quando a investigação do conteúdo é feita por relato, pois as mães têm maior probabilidade de recordar procedimentos do que aconselhamento recebido. É possível também que a mulher não recorde ter recebido um procedimento porque não reconhece o procedimento como tal 35.

Ao se analisar o resultado dos estudos que avaliaram o efeito do pré-natal sobre desfechos perinatais, é necessário que se entenda a relação dos vieses envolvidos e que distorcem as associações encontradas; que se avalie criticamente o pressuposto assumido, quando da seleção do indicador; e que se conheça a acurácia do indicador para medir a adequação do cuidado pré-natal 35,41 .

Em primeiro lugar, destaca-se o viés de auto-seleção dos estudos observacionais, pois as mulheres que decidem pela busca do cuidado e iniciam o pré-natal mais cedo são diferentes em importantes aspectos daquelas que o retardam, implicando em forte probabilidade de causalidade reversa.

Em segundo lugar, a disponibilidade e a acurácia das informações e o uso de dados secundários, não gerados para fins de pesquisa, e geralmente utilizados, podem limitar a qualidade dos estudos. Na maioria dos estudos, os dados são extraídos de certificados de nascimento, por vezes com problemas de acurácia e com proporção considerável de informação ignorada, o que freqüentemente leva a erro de medida e conseqüente erro de classificação. Sabe-se que vários estudos comparando certificados de nascimento com registros clínicos encontraram um número mais alto de visitas de pré-natal auto-relatadas nos primeiros do que nos últimos 42, e que esses documentos não dispõem de informações qualitativas.

Finalmente, o indicador de adequação do pré-natal escolhido, o tratamento dado e as informações ignoradas na composição das categorias também podem influir nos resultados alcançados 17,42.

Em resumo, os achados desta revisão mostram que a influência da atenção pré-natal sobre o peso ao nascer não é inequívoca, principalmente devido ao efeito do viés de auto-seleção. Estudos randomizados são necessários para definir, com maior grau de certeza, o impacto do pré-natal sobre o peso ao nascer e outros desfechos da gestação humana. 


\section{Resumo}

Esta foi uma revisão sistemática da literatura de publicações em que o pré-natal foi investigado com uma das variáveis preditoras do peso ao nascer. Os bancos de dados MEDLINE, Cochrane Library e SciELO foram rastreados usando-se a combinação dos seguintes descritores: "prenatal care", "antenatal care", "quality", "adequacy", "birthweight", $e$ "low birthweight". Foram localizados 25 estudos: 17 transversais, quatro coortes, três caso-controle e um ensaio randomizado. Os indicadores de adequação empregados foram os de utilização (quantitativos) e os de conteúdo do cuidado (de processo ou qualitativos). A maioria dos autores aplicou indicadores de utilização, principalmente o Índice de Kessner e o Adequacy of Prenatal Care Utilization Index. Somente dois estudos usaram critérios qualitativos. De modo geral, os estudos transversais detectaram efeito protetor do pré-natal sobre o baixo peso ao nascer, enquanto que os resultados de investigações com outros desenhos foram conflitantes. Os achados desta revisão evidenciam que o impacto do pré-natal sobre o peso ao nascer não é inequívoco, principalmente devido ao efeito do viés de auto-seleção. Há a necessidade de realização de ensaios randomizados para esclarecer essa relação.

Cuidado Pré-natal; Peso ao Nascer; Qualidade; Literatura de Revisão

\section{Colaboradores}

D. S. Silveira contribuiu para concepção e desenho do estudo; aquisição dos dados, sua análise e interpretação; redação do artigo para submissão à publicação; revisão crítica do conteúdo do artigo após comentários do corpo editorial da revista a qual foi submetido; redação e aprovação final do artigo após correções. I. S. Santos contribuiu para concepção e desenho do estudo; análise e interpretação dados; redação do artigo para submissão à publicação; revisão crítica do conteúdo do artigo após comentários do corpo editorial da revista a qual foi submetido; redação e aprovação final do artigo após correções.

\section{Referências}

1. Gazmarian JA, Arrigton MPA, Bailey CM, Schwartz MSW, Koplan JP. Prenatal care for low-income women enrolled in a managed-care organization. Obstet Gynecol 1999; 94:177-84.

2. Dye TD, Wojtowcz MA. Organizational variation, satisfaction, and women's time investment in prenatal acre. Paediatr Perinat Epidemiol 1999; 13:158-69.

3. Enkin M. Randomized controlled trial in the evaluation of antenatal care. Int J Technol Assess Health Care 1992; 8 Suppl 1:40-5.

4. Peabody JW, Gertler PJ, Leibowitz A. The policy implications of better structure and process on birth outcomes in Jamaica. Health Policy 1998; 43:1-13.

5. Kestler E. Wanted: better care for pregnant women. World Health Forum 1993; 14:356-9.

6. Munjanja SP, Lindmark G, Nyström L. Randomised controlled trial of a reduced-visits programme of antenatal care in Harare, Zimbabwe. Lancet 1996; 348:364-9

7. Villar J, Khan-Neelofur D. Patterns of routine care for low-risk pregnancy. Patterns of routine antenatal care for low-risk pregnancy (Cochrane Review). In: The Cochrane Library, Issue 4, 1999. Oxford: Update Software.

8. Petitti DB, Hiatt RA, Chin V, Croughan-Minihane M. An outcome evaluation of the content and quality of prenatal care. Birth 1991; 18:21-5.

9. Kogan MD, Martin JA, Alexander GR, Kotelchuck M, Ventura SJ, Frigoletto F. The changing pattern of prenatal care utilization in the United States, 1981-1995, using different prenatal care indices. JAMA 1998; 279:1623-8.

10. Deslandes SF. Concepções em pesquisa social: articulações com o campo da avaliação em serviços de saúde. Cad Saúde Pública 1997; 13:103-7.

11. Hansell MJ. Sociodemographic factors and the quality of prenatal care. Am J Public Health 1991; 81:1023-8.

12. Kotelchuck M. An evaluation of the Kessner Adequacy of Prenatal Care Index and a proposed Adequacy of Prenatal Care Utilization Index. Am J Public Health 1994; 84:1414-20.

13. Kotelchuck M. The Adequacy of Prenatal Care Utilization Index: its US distribution and association with low birthweight. Am J Public Health 1994; 84:1486-9.

14. Brett KM, Schoendorf KC, Kiely JL. Differences between black and white women in the use of prenatal care technologies. Am J Obstet Gynecol 1994; 170:41-6.

15. Ogunbekun I, Adeyi O, Wouters A, Morrow RH. Costs and financing of improvements in the quality of maternal health services through the Bamako iniciative in Nigeria. Health Policy Plan 1996; 11:369-84.

16. Langer A, Nigenda G, Romero M, Rojas G, Kuchaisit C, Al-Osimi M, Orozco E. Conceptual basis and methodology for the evaluation of women's and providers' perception of the quality of antenatal care in the WHO Antenatal Care Randomized Controlled Trial. Paediatr Perinat Epidemiol 1998; 12 Suppl 2:98-115. 
17. Alexander GR, Tompkins ME, Petersen DJ, Weiss J. Sources of bias in prenatal care utilization indices: implications for evaluating the medical expansion. Am J Public Health 1991; 81:1013-6.

18. McDonagh $\mathrm{M}$. Is antenatal care effective in reducing maternal morbidity and mortality? Health Policy Plan 1996; 11:1-15.

19. Katz SJ, Armstrong RW, LoGerfo JP. The adequacy of prenatal care and incidence of low birthweight among the poor in Washington State and British Columbia. Am J Public Health 1994; 84:986-91.

20. Delgado-Rodríguez M, Gómez-Olmedo M, BuenoCavanilhas A, Gálvez-Vargas RA. Comparison of two indices of adequacy of prenatal care utilization. Epidemiology 1996; 7:648-50.

21. Haas JS, Orav EJ, Goldman L. The relationship between physicians' qualifications and experience and the adequacy of prenatal care and low birthweight. Am J Public Health 1995; 85:1087-91.

22. Bradley PJ, Martin J. Issues inherent in measuring the impact of care coordination on pregnancy. Public Health Nurs 1996; 13:276-85.

23. Ramirez-Zetina $M$, Richardson V, Ávila H, Caraveo VE, Salomón RE, Jimenéz-Cruz A. La atención prenatal en la ciudad fronteriza de Tijuana, México. Rev Panam Salud Publica 2000; 2:97-101.

24. Poland ML, Ager JW, Olson KL, Sokol RJ. Quality of prenatal care; selected social, behavioral, and biomedical factors; and birth weight. Obstet Gynecol 1990; 75:607-8.

25. Collins JW, David RJ. Differences in neonatal mortality by race, income, and prenatal care. Ethn Dis 1992; 2:18-26.

26. Barros H, Tavares M, Rodrigues T. Role of prenatal care in preterm, birth and low birthweight in Portugal. J Public Health Med 1996; 18:321-8.

27. Markson LE, Turner BJ, Houchens R, Silverman NS, Cosler L, Takyi BK. Association of maternal HIV infection with low birth weight. J Acquir Immune Defic Syndr Hum Retrovitol 1996; 13:227-34.

28. Turner BJ, McKee LJ, Silverman NS, Hauck WW, Fanning TR, Markson LE. Prenatal care and birth outcome of a cohort of HIV-infected women. J Acquir Immune Defic Syndr Hum Retrovitol 1996; 12:259-67.

29. Raine T, Powell S, Krohn MA. The risk of repeating low birth weight and the role of prenatal care. Obstet Gynecol 1994; 84:485-9.

30. Shohan-Vardi I, Levy E, Belmaker I, Mazor M, Goldstein D. Utilization of prenatal services and birth outcomes: a community-based study. Paediatr Perinat Epidemiol 1997; 11:271-86.

31. Newschaffer CJ, Cocroft J, Hauck WW, Fanning T, Turner BJ. Improved birth outcomes associated with enhanced Medicaid prenatal care in drugusing women infected with the Human Immunodeficiency Virus. Obstet Gynecol 1998; 91:885-91.

32. Krueger PM, Scholl TO. Adequacy of prenatal care and pregnancy outcome. J Am Osteopath Assoc 2000; 100:485-92.

33. Wright EA. Low birthweight in the plateau region of Nigeria. East Afr Med J 1990; 7:894-9.
34. Turner BJ, Cocroft J, Newschaffer CJ, Hauck WW, Fanning TR, Berlin M. Sources of prenatal care data and their association with birth outcomes of HIV-infected women. Am J Public Health 2000; 90:118-21.

35. Kogan MD, Alexander GR, Kotelchuck N, Nagey DA, Jack BW. Comparing mothers' reports on the content of prenatal care received with recommended national guidelines for care. Public Health Rep 1994; 109:637-46.

36. Last JM. A dictionary of epidemiology. New York: Oxford University Press; 1995.

37. Rothman KJ, Greenland S. Modern epidemiology. 2nd Ed. Philadelphia: Lippincott-Raven; 1998.

38. Moore P, Hepworth JT. Use of perinatal and infanth health services by Mexican-American Medicaid enrolles. JAMA 1994; 272:297-304.

39. Osis MJD, Hardy E, Faúndes A, Alves G. Fatores associados à assistência pré-natal entre mulheres de baixa renda no Estado de São Paulo, Brasil. Rev Saúde Pública 27:49-53.

40. McDermott J, Drews C, Green D, Berg C. Evaluation of prenatal care information on birth certificates. Paediatr Perinat Epidemiol 1997; 11:105-21.

41. Kotelchuck M. Adequacy of prenatal care utilization. Epidemiology 1997; 8:602-3.

42. Griffin JF, Hogan JW, Buechner JS, Leddy TM. The effect of a Medicaid Managed Care Program on the adequacy of prenatal care utilization in Rhode Island. Am J Public Health 1999; 89:497-501.

43. Alexy B, Nichols B, Heverly MA, Garzon L. Prenatal factors and birth outcomes in the public health service: a rural/urban comparison. Res Nurs Health 1997; 20:61-70.

44. Balcazar H, Harter J, Cole G. The effects of prenatal care utilization and maternal risk factors on pregnancy outcome between Mexican Americans and non-Hispanic whites. J Natl Med Assoc 1993; 85:195-202.

45. Brabin L, Verhoeff FH, Kazembe P, Brabin BJ, Broadheaded R. Improving antenatal care for pregnant adolescents in southern Malawi. Acta Obstet Gynecol Scand 1998; 77:402-9.

46. McDuffie RS, Beck A, Bischoff K, Cross J, Orleans $M$. Effect of frequency of prenatal care visits on perinatal outcome among low-risk women. JAMA 1996; 275:847-51.

47. Petersen DJ, Alexander GR, D'Ascoli P, Oswald J. Prenatal care utilization in Minnesota. Patterns of concern, areas for improvement. Minn Med 1994; 77:41-5.

48. Silva AAM, Lamy Filho F, Alves MTSSB, Coimbra LC, Bettiol H, Barbieri MA. Risk factors for low birthweight in north-east Brazil: the role of caesarean section. Paediatr Perinat Epidemiol 2001; 15:257-64.

Recebido em 01/Out/2003

Versão final reapresentada em 22/Mar/2004 Aprovado em 27/Abr/2004 